\title{
Unstructured hexahedral mesh generation of complex vascular trees using a multi-block grid-based approach
}

\section{Introduction}

2 
tion of the cross section for creating (block) structured hexahedral grids of tubular structures (Berthier et al., 2002; Gao et al., 2006; Shimogonya et al., 2009) and bifurcations (Long et al., 1998; Younis et al., 2007) have been proposed in the last decade. This led to the development of robust and automated strategies where the sweeping is based on a skeleton (Antiga et al., 2002), on the solution of a thermal conduction problem (Verma et al., 2005) or on geometrical features and operator choices using a graphical user interface (De Santis et al., 2011a). Decomposition templates for various branching configurations have been proposed (Zhang et al., 2007). However, a configuration with a single bifurcation may already lead to skewed elements at this bifurcation region, limiting the use of these sweeping techniques to 'simple' bifurcations only.

When geometries comprise multiple branching points or n-furcations, hexahedral meshing strategies based on mapping can be used. These strategies combine a preliminary generated multi-block structure with a volumetric block refinement to create the grid points. A body-fitted grid is then generated by mapping the volume mesh to the volume of interest and by mapping the border mesh onto the surface of the image-based geometry (Grosland et al., 2009). A boundary layer grid can be included by introducing building blocks with a so-called 'butterfly' pattern, in which four blocks surround a fifth block at the core (Zhang et al., 2000). This approach can be semiautomated by building multi-block structures inside and outside the vessel geometry as demonstrated by De Santis et al. (2011b). However, current procedures for generating adequate multi-block structures are still not fully 
automated or lack robustness, and manual interventions are required to correct self-intersecting or low quality blocks (De Santis et al., 2011b; Ramme et al., 2011, 2012) leading to intensive and extensive operator tasks. Moreover, block corrections to assure a proper mapping in high-curvature regions of complex geometries - by pursuing small variations in the local geodesic distance between the geometry and the multi-block structure - typically deteriorate the quality of the blocks and the cells of the final mesh.

The rationale of this work is to address these shortcomings by providing a robust and high-quality hexahedral meshing procedure that allows automated mesh generation of complex vascular trees and is able to handle any combination of vessel segments, side branches, stenoses and aneurysms. This paper presents a novel procedure for auto-generating an interconnection of hexahedral blocks (Section 2.1). The resulting multi-block structure is further processed and used as input for a grid-based method to generate an unstructured hexahedral grid of the fluid domain (Section 2.2). Finally, the performance of the generated grids is analyzed in two numerical examples (Section 3).

\section{Materials and methods}

The methods proposed in this paper were implemented in a pyFormex framework combined with output from a geometrical analysis using the vmtk software. 


\subsection{Multi-block structure generation}

In this section a strategy is proposed to generate a hexahedral multi-block structure of a treelike network by expressing the branching topology in terms of 'parent vessels' (Section 2.1.1), followed by the construction and alignment of vessel-enclosing squares (Section 2.1.2) and their interconnection into a set of hexahedral blocks (Section 2.1.3).

\subsubsection{Branching topology}

Given the in- and outlets of the geometry, a centerline with a constant prescribed distance $d_{0}$ between the centerline nodes is computed, together with the radii of the maximum inscribed spheres, and the distances between the surface geometry and the centerline (Antiga et al., 2008). Each set of centerline nodes, corresponding to a vessel segment between two consecutive branching points or between a branching point and an inlet or outlet, is further referred to as a 'branch'.

The connectivity between the branches is extracted from the centerline data and used to define the complete branching topology in terms of 'parent vessels', Figure 1(a). Starting with the branch at the inlet, consecutive branches are gathered into one (non-bifurcating) parent vessel, the so-called zeroth order parent vessel. This is done such that for every branching point the angle between the direction vector of the incoming and outgoing parent branch is minimal. The remaining child branches are listed per branching point using a FIFO-buffer, and set as start branches to retrieve the first order parent vessels. This procedure is repeated as long as the FIFO-buffer is filled with start branch labels of next order parent vessels. 


\subsubsection{Square alignment}

For each branch, squares are constructed of which the centroids are located at the centerline nodes, of which the normals are tangent to centerline and of which one side is oriented parallel to the local transversal direction vector, which gets calculated such that a smooth transition of the squares is realized in every branch and across the branching points, Figure 1(b). Therefore, a bifurcation normal is constructed at every branching point, normal to the plane defined by the direction vectors of the outgoing parent branch and a child branch. Then, each bifurcation normal is rotated in steps of $\frac{\Pi}{2}$ radians around the direction vector of the outgoing parent branch until the deflection angle with the upstream bifurcation normal (of the same parent vessel) is minimal. The resulting bifurcation normals are projected onto the planes perpendicular to the direction vectors of the surrounding branches, Figure 1(a). The projected normals are interpolated along the centerline, $\vec{n}_{p}^{m}$, to compute the local transversal direction vector, $\vec{e}_{t}^{m}$, at every centerline node $m$, Equation (1), with $\vec{e}_{a}^{m}$ the local axial direction vector and $N_{c}^{b}$ the number of centerline nodes at branch $b$.

$$
\begin{aligned}
& \vec{n}_{p}^{m}=\vec{n}_{p}^{0}+\frac{m}{N_{c}^{b}-1}\left(\vec{n}_{p}^{N_{c}^{b}-1}-\vec{n}_{p}^{0}\right), \quad m \in\left[0, N_{c}^{b}-1\right] \\
& \vec{e}_{t}^{m}= \begin{cases}\frac{\vec{n}_{p}^{m} \times \vec{e}_{a}^{m}}{\left\|\vec{n}_{p}^{m} \times \vec{e}_{a}^{m}\right\|} & , \quad \vec{n}_{p}^{m} \times \vec{e}_{a}^{m} \neq \overrightarrow{0} \\
\frac{\vec{e}_{t}^{m-1}-\left(\vec{e}_{t}^{m-1} \cdot \vec{e}_{a}^{m}\right) \cdot \vec{e}_{a}^{m}}{\left\|\vec{e}_{t}^{m-1}-\left(\vec{e}_{t}^{m-1} \cdot \vec{e}_{a}^{m}\right) \cdot \vec{e}_{a}^{m}\right\|} \quad, \quad \vec{n}_{p}^{m} \times \vec{e}_{a}^{m}=\overrightarrow{0}\end{cases}
\end{aligned}
$$

108

Each square gets an edge length relative to the local diameter of the maximum inscribed sphere with a scaling factor $f_{s}>1$ to radially enclose 
the surface geometry (De Santis et al., 2011b).

\subsubsection{Block generation and interconnection}

The squares are auto-connected into a multi-block structure, based on the branching topology as defined in Section 2.1.1 (Figure 1(c)). Note that there exists only one parent vessel of the zeroth order $(i=0)$. A block structure for this parent vessel is built by interconnecting the consecutive squares of its branches. First, a curved block structure is generated for its first branch. As long as this branch is not the last branch of the parent vessel, this step is followed by the generation of a curved block structure for this next (downstream) branch and a branch connection block, connecting the curved block structures of both branches. Similar procedures are used to build the parent vessel block structures of order $i>0$ with the difference that additional connection blocks, towards the corresponding parent vessels of order $i-1$, are built too. These vessel connection blocks are constructed by extracting branch connection blocks from a FIFO-buffer, which got filled when building the parent vessel block structures of order $i-1$.

The generated multi-block structure is axially smoothed - keeping the faces at the inlet, the outlets and the branch connection blocks in place - to avoid self-intersection and to improve the vessel connection blocks.

\subsection{Unstructured hexahedral mesh generation}

In this section, a refined multi-block structure (Section 2.2.1) and a radially compressed surface mesh of the vascular tree are provided as input to the well-known grid-based method (Ho-Le, 1988). The result is an unstruc-

tured set of (centerline aligned) hexahedral volume elements for the core of 
the fluid domain (Section 2.2.2). This unstructured hex-core resembles the volume of the original geometry well and allows the creation of a high-quality boundary layer grid between the interface of the hex-core and the original surface mesh (Section 2.2.3).

\subsubsection{Multi-block structure refinement}

The multi-block structure from Section 2.1 is refined by introducing a grid in every block, Figure 2(a). The resulting grids are the outcome of a refinement procedure which is based on two parameters: the axial edge refinement factor, $f_{\text {edge, } a}(\in \mathbb{N})$, and the transversal edge refinement factor, $f_{\text {edge,t }}(\in \mathbb{N})$. The first subdivides the edges in the direction of the centerline, where the latter defines the refinement of the edges in a plane perpendicular to the centerline and at all the edges of the branch connection blocks (where axial and transversal directions are not unambiguously defined). $f_{\text {edge, } a}$ may vary throughout the model, where $f_{\text {edge,t }}$ is kept constant to avoid a nonconformal mesh at the interface regions between two refined, consecutive blocks.

\subsubsection{The multi-block grid-based method}

In the grid-based method, an initial Cartesian grid is overlaid on the input surface geometry and the grid cells falling outside the computational domain are removed. To obtain a body-fitted grid, the stair-step surface mesh is projected onto the bounding surface of the input geometry (Schneiders, 1996). A mesh resulting from the grid-based method is unstructured and consists of hexahedral volume elements with an excellent cell quality in the object interior and near the boundaries that are parallel to one of 
the coordinate planes of the initial grid. A drawback of this method is the generation of highly skewed cells near the boundaries which are not parallel to one of the coordinate planes. The quality of these cells largely depends on the orientation of the initial grid. Therefore, the authors apply the gridbased method onto a refined multi-block structure and not onto a Cartesian grid. This new approach is further denoted by the multi-block grid-based method. The multi-block structure comprises curved block structures which are aligned with the centerline and take into account the local vessel radius. During the projection phase this results in stretching the cells towards the boundary rather than skewing them. To allow a proper projection, the volume of the remaining cells - further denoted as the hex-core (Figure 2(b)) is corrected by removing all cells with non-manifold edges. Then, the stairstep surface mesh of the hex-core is smoothed and projected onto the input surface geometry to obtain a body-fitted hex-core, Figure 2(c).

\subsubsection{Boundary layer grid generation}

The cell quality at the boundary can be further improved by inserting buffer layers (Tchon et al., 1997; Kovalev, 2005; Shepherd et al., 2006). In this work a similar effect is obtained by performing the grid-based method onto an input surface geometry of which the interface mesh (i.e. the surface mesh excluding the faces at the in- and outlets) is a radially compressed isomorphism of the original (lumen-wall) interface mesh. This allows the creation of a boundary layer grid with a controllable thickness, e.g. by setting the distance between the original and the compressed interface as a function of the local distance to centerline, Figure 3(a), combined with a threshold to limit the maximum thickness of the boundary layer grid (for instance in 
aneurysm regions).

The body-fitting process of the grid-based method projects the interface nodes of the hex-core onto the compressed interface mesh. Isoparametric coordinates for each of these nodes are computed with respect to the face where they are projected onto. As the compressed interface mesh holds the same mesh topology as the original interface mesh, isoparametric coordinate transformations map the hex-core interface onto this original interface mesh. The mapped nodes are then connected to the corresponding nodes of the isomorphic hex-core interface, generating one layer of hexahedral cells which can be further refined radially to obtain multiple layers at the boundary layer. While keeping the border mesh in place, a final Laplacian smoothing is performed to improve the cell quality at the transition region between the hex-core and the boundary layer grid, Figure 3(b).

\section{Numerical examples}

In this section, numerical analyses of two applications illustrate the efficacy of the unstructured hexahedral grids.

\subsection{CFD model of an abdominal mouse aorta}

\subsubsection{Grid sensitivity analysis}

A grid sensitivity analysis is performed for a steady state CFD simulation at peak systole of an abdominal mouse aorta, using the flow solver Fluent (Ansys). The geometry (Figure 4(b)) and the boundary conditions (the peak systolic inflow of case $A A 7$ combined with the mean outflow fractions) were taken from Trachet et al. (2011). 
Pressure drops and velocities were analyzed along the centerline for multiple hexahedral grids with increasing mesh densities. Their mean error and maximum error with respect to the reference grid are presented in Table 1, relative to the total pressure drop $\Delta p=6.822 \mathrm{mmHg}$ and the maximum change in velocity along the centerline $\Delta v=32.310 \frac{\mathrm{cm}}{\mathrm{s}}$. To allow an adequate comparison of the wall shear stress (WSS) for different grids, it is evaluated on a global level by considering the surface area enclosed by a WSS iso-contour in function of WSS, $A_{i s o}$ (wss), normalized by the total surface area, $A_{t o t}$. The mean and maximum errors with respect to the reference grid are presented in Table 1. As the errors are small, the derivative of this function is visualized in Figure 4(a) for values larger than 5\% of the maximum surface area change. To evaluate WSS on a local level the iso-contour of $10.8 \mathrm{~Pa}$, which corresponds to both a high WSS value and a high change in WSS surface area, is visualized in Figure 4(b) for different mesh densities in the region distal to the coeliac artery and proximal to the trifurcation.

Table 1 shows small errors for all three flow variables and a converging trend. $53 \mathrm{k}, 98 \mathrm{k}$ and $798 \mathrm{k}$ cells are required in the fluid domain to obtain a maximum error of about $1 \%$ for respectively the pressure along the centerline, the global change in WSS surface area and the velocity along the centerline. Moreover, Figure 4(b) shows only a small error and a converging trend for the WSS surface area on a local level.

The efficacy of hexahedral grids becomes clear when comparing the velocity along the centerline to results obtained for a grid sensitivity analysis involving tetrahedral grids (with a prismatic boundary layer grid near the wall), Figure 5, where 201k hexahedral cells provide the same accuracy as $11870 \mathrm{k}$ 
tetrahedral cells.

\subsubsection{Multi-block structure extension}

In more complex geometries, high-curvature regions may lead to lowquality cells. The presented meshing strategy can be extended by additional block-structures. For example, consider the aneurismatic mouse aorta of Figure 6(a). The multi-block structure is constructed after adapting the, in Section 2.1.1 defined, branching topology as suggested in Figure 6(b). Then, based on the bounding box of the aneurysm and trifurcation region, an additional connection block structure is generated to replace the branch with label 1 and the corresponding branch connection blocks, Figure 6(c). Detailed views of the fluid mesh and an equiangle skewness histogram are provided in Figure 6(d).

\subsection{Local grid refinement in an FSI model of aortic coarctation}

This example analyses the pressure drop along an aorta with aortic coarctation, which is a congenital disease characterized by a narrowing of the upper descending aorta. The pressure difference between the ascending and descending aorta is the most important indication of aortic coarctation. Because this pressure drop is difficult to assess in a non-invasive way, its simulation is of high clinical relevance. As simulations with rigid walls fail to capture some physiological patterns, the fluid-structure interaction (FSI) between the blood flow and the deformation of the arterial wall was taken into account. To obtain an accurate calculation of the stress on the fluid-structure interface, the flow equations were solved in the Arbitrary Lagrangian-Eulerian formulation on a deforming mesh, using Fluent (Ansys). The structural equations 
were solved in a Lagrangian frame, using Abaqus/Standard (Simulia). The solvers were strongly coupled with the IQN-ILS technique (Degroote et al., 2009).

The geometrical model was obtained from MRI images of a healthy $39 y$ old male volunteer. The functional impact of aortic coarctation was modeled by including a stenosed region with a length of $1 \mathrm{~cm}(\mathrm{~L})$ and a coarctation index $\left(\mathrm{CI}=\mathrm{D}_{\text {coa }} / \mathrm{D}_{\text {desc }}\right)$ of 0.5 , Figure $8(\mathrm{a})$. Flow rates were measured with phase-contrast MRI and imposed as physiological boundary conditions at the ascending aorta and the three side branches. At the descending aorta, a three-element Windkessel model was implemented $\left(Z=0.08 \frac{\mathrm{mmHg}}{\mathrm{ml} / \mathrm{s}}, R=\right.$ $\left.1.024 \frac{\mathrm{mmHg}}{\mathrm{ml} / \mathrm{s}}, C=2.0 \frac{\mathrm{ml}}{\mathrm{mmHg}}\right)$, in which the parameters of the model were defined such that physiological pressure variations were retrieved. Blood was modeled as a Newtonian fluid (viscosity $3 \mathrm{mPas}$, density $1050 \frac{\mathrm{kg}}{\mathrm{m}^{3}}$ ). As a nonconformal mesh is allowed at the fluid-structure interface, the quadrilateral interface can be simplified, Figure 7(a). This improves the quality of the mesh for the arterial wall which is generated by radially expanding the quadrilateral interface into a hexahedral mesh with multiple layers (diameter to thickness ratio: 10\%). Only radial displacement was allowed at the in-and outlet boundaries of the solid domain and the material behavior of the aortic tissue was described using a polynomial hyperelastic model (hyperelastic constants: $\left.C_{10}=18.9 \mathrm{kPa}, C_{01}=2.75 \mathrm{kPa}, C_{20}=400 \mathrm{kPa}, C_{11}=847.2 \mathrm{kPa}\right)$.

For a constant square scaling factor, Figure 7(b), four different meshes were constructed with an increasing cell density (R1, R2, R3, R4). The number of cells are depicted in Table 2, together with the calculation time required to compute one cardiac cycle (on two 10-core Intel Xeon E5-2680v2 
processors). As FSI simulations are computationally expensive and time consuming, it is important to use a computational grid for which accurate results are obtained with a limited number of cells. To illustrate how the proposed meshing strategy is able to provide an adaptively refined grid, a fifth mesh is constructed (R5) by locally adapting $f_{e d g e, a}$ and $f_{s}$, Figure 7 (c). The resulting fluid mesh has, compared to the finest mesh (R4), a higher mesh density in the coarctation zone, but a coarser grid proximal to the stenosis and in the lower part of the descending aorta.

The results of the grid refinement study are shown in Figure 8(b), depicting the pressure evolution along the centerline of the aorta at peak systole, and in Table 2, showing the mean error of the pressure evolution in different cross sections. These errors are defined with respect to the reference grid R4 and relative to the pressure amplitude in the corresponding cross section. From the results in Table 2, it can be seen that even for meshes with a low cell density, the mean errors proximal, halfway and distal to the coarctation zone remain low $(<2 \%)$. When comparing the locally refined grid R5 with the uniformly refined grids R3 and R4, an important reduction in computation time is gained $(23 \mathrm{~h} 38 \mathrm{~min}$ per cardiac cycle versus $30 \mathrm{~h} 33 \mathrm{~min}$ and $40 \mathrm{~h}$ $38 \mathrm{~min})$ without a loss in accuracy. The mean error obtained with the mesh R5 stays below $1.15 \%$ and comparable errors are found as for the mesh R3.

\section{Conclusion}

This work proposes a novel strategy to generate unstructured hexahedral grids for the fluid domain of complex treelike structures. By only providing the grid refinement parameters, a multi-block structure is generated, refined, 
and used - together with a radially compressed surface mesh of the geometry of interest - as input for the grid-based method. The resulting hex-core is extended with a boundary layer grid to generate a mesh for the fluid domain with high-quality cells, even in regions of high curvature. The methodology allows local refinements in both the axial direction and the cross sections, and is able to handle aneurysms by extending the multi-block structure with additional blocks. A grid dependency study, for a steady state CFD simulation of an abdominal mouse aorta, shows a fast converging trend for the pressure gradient, the velocity and the wall shear stress. The accuracy is superior compared to tetrahedral grids with a prismatic boundary layer grid, and for the same accuracy a hexahedral mesh appears to reduce the number of cells by a factor $0.1-0.01$. Finally, a numerical example of aortic coarctation shows the applicability of the meshing strategy to complex geometries in FSI models and the efficacy of local grid refinements in reducing the computation time without accuracy loss.

\section{Acknowledgements}

This research was funded by the Special Research Fund of the Ghent University (BOF10/GOA/005).

\section{Conflict of interest statement}

The authors report that there is no conflict of interest regarding this study. 


\section{References}

Antiga, L., Ene-Iordache, B., Caverni, L., Cornalba, G.P., , Remuzzi, A., 2002. Geometric reconstruction for computational mesh generation of arterial bifurcations from CT angiography. Computerized Medical Imaging and Graphics 26, $227-235$.

Antiga, L., Ene-Iordache, B., Remuzzi, A., 2003. Computational geometry for patient-specific reconstruction and meshing of blood vessels from MR and CT angiography. IEEE Transactions on Medical Imaging 22, 674-684.

Antiga, L., Piccinelli, M., Botti, L., Ene-Iordache, B., Remuzzi, A., Steinman, D.A., 2008. An image-based modeling framework for patient-specific computational hemodynamics. Medical \& Biological Engineering \& Computing 46, 1097-1112.

Berthier, B., Bouzerar, R., Legallais, C., 2002. Blood flow patterns in an anatomically realistic coronary vessel: influence of three different reconstruction methods. Journal of Biomechanics 35, 1347 - 1356.

Biswas, R., Strawn, R.C., 1998. Tetrahedral and hexahedral mesh adaptation for CFD problems. Applied Numerical Mathematics 26, 135 - 151.

De Santis, G., De Beule, M., Segers, P., Verdonck, P., Verhegghe, B., 2011a. Patient-specific computational haemodynamics: generation of structured and conformal hexahedral meshes from triangulated surfaces of vascular bifurcations. Computer Methods in Biomechanics and Biomedical Engineering 14, 797-802. 
De Santis, G., De Beule, M., Van Canneyt, K., Segers, P., Verdonck, P., Verhegghe, B., 2011b. Full-hexahedral structured meshing for image-based computational vascular modeling. Medical Engineering \& Physics 33, 1318 $-1325$.

De Santis, G., Mortier, P., De Beule, M., Segers, P., Verdonck, P., Verhegghe, B., 2010. Patient-specific computational fluid dynamics: structured mesh generation from coronary angiography. Medical \& Biological Engineering \& Computing 48, 371-380.

Degroote, J., Bathe, K., Vierendeels, J., 2009. Performance of a new partitioned procedure versus a monolithic procedure in fluid-structure interaction. Computers and Structures 87, 793-801.

Gao, F., Guo, Z., Sakamoto, M., Matsuzawa, T., 2006. Fluid-structure interaction within a layered aortic arch model. J Biol Phys 32, 435-54.

Grosland, N.M., Shivanna, K.H., Magnotta, V.A., Kallemeyn, N.A., DeVries, N.A., Tadepalli, S.C., Lisle, C., 2009. IA-FEMesh: An open-source, interactive, multiblock approach to anatomic finite element model development. Computer Methods and Programs in Biomedicine 94, 96 - 107.

Ho-Le, K., 1988. Finite element mesh generation methods: A review and classification. Comput. Aided Des. 20, 27-38.

Kovalev, K., 2005. Unstructured Hexahedral Non-conformal Mesh Generation (PhD Thesis). Ph.D. thesis. Vrije Universiteit Brussel.

Liu, Y., Pekkan, K., Jones, S.C., Yoganathan, A.P., 2004. The effects of different mesh generation methods on computational fluid dynamic analysis 
and power loss assessment in total cavopulmonary connection. Journal of Biomechanical Engineering 126, 594-603.

Long, Q., Xu, X., Collins, M.W., Bourne, M., Griffith, T., 1998. Magnetic resonance image processing and structured grid generation of a human abdominal bifurcation. Computer Methods and Programs in Biomedicine $56,249-259$.

Longest, P.W., Vinchurkar, S., 2007. Effects of mesh style and grid convergence on particle deposition in bifurcating airway models with comparisons to experimental data. Medical Engineering \& Physics 29, 350 - 366.

Ramme, A.J., Shivanna, K.H., Criswell, A.J., Kallemeyn, N.A., Magnotta, V.A., Grosland, N.M., 2012. Growing multiblock structures: a semiautomated approach to block placement for multiblock hexahedral meshing. Computer Methods in Biomechanics and Biomedical Engineering 15, 1043-1052.

Ramme, A.J., Shivanna, K.H., Magnotta, V.A., Grosland, N.M., 2011. Gaussian curvature analysis allows for automatic block placement in multi-block hexahedral meshing. Computer Methods in Biomechanics and Biomedical Engineering 14, 893-904.

Schneiders, R., 1996. A grid-based algorithm for the generation of hexahedral element meshes. Engineering with Computers 12, 168-177.

Shepherd, J.F., Tuttle, C.J., Silva, C.T., Zhang, Y., 2006. Quality Improvement and Feature Capture in Hexahedral Meshes. Technical Report Tech- 
nical Report UUSCI-2006-029. SCI Institute, University of Utah. Salt Lake City, UT, USA.

Shimogonya, Y., Ishikawa, T., Imai, Y., Matsuki, N., Yamaguchi, T., 2009. Can temporal fluctuation in spatial wall shear stress gradient initiate a cerebral aneurysm? A proposed novel hemodynamic index, the gradient oscillatory number (GON). Journal of Biomechanics 42, 550 - 554 .

Tchon, K.F., Hirsch, C., Schneiders, R., 1997. Octree-based Hexahedral Mesh Generation for Viscous Flow Simulations. Technical Report A9732470. American Institute of Aeronautics and Astronautics.

Trachet, B., Bols, J., De Santis, G., Vandenberghe, S., Loeys, B., Segers, P., 2011. The impact of simplified boundary conditions and aortic arch inclusion on CFD simulations in the mouse aorta: a comparison with mouse-specific reference data. Journal of Biomechanical Engineering 133, 121006(13).

Verma, C.S., Fischer, P.F., Lee, S., Loth, F., 2005. An all-hex meshing strategy for bifurcation geometries in vascular flow simulation, in: In 14th International Meshing Roundtable, pp. 363 - 375.

Vinchurkar, S., Longest, P.W., 2008. Evaluation of hexahedral, prismatic and hybrid mesh styles for simulating respiratory aerosol dynamics. Computers \& Fluids 37, $317-331$.

Younis, B., Spring, S., Neumann, S.O., Weigand, B., 2007. Simulation of flow in an exact replica of a diseased human carotid artery. Applied Mathematical Modelling 31, 2599 - 2609. 
Zhang, Y., Bazilevs, Y., Goswami, S., Bajaj, C.L., Hughes, T.J.R., 2007. Patient-specific vascular NURBS modeling for isogeometric analysis of blood flow. Computer Methods in Applied Mechanics and Engineering $196,2943-2959$.

Zhang, Z., Kleinstreuer, C., Kim, C.S., 2000. Flow structure and particle transport in a triple bifurcation airway model. Journal of Fluids Engineering 123, 320-330. 


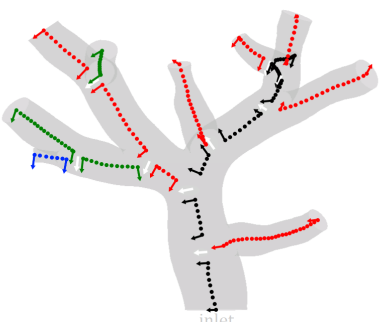

(a)

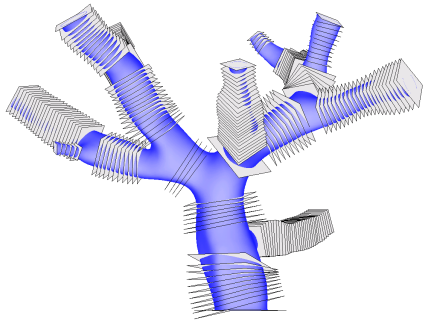

(b)

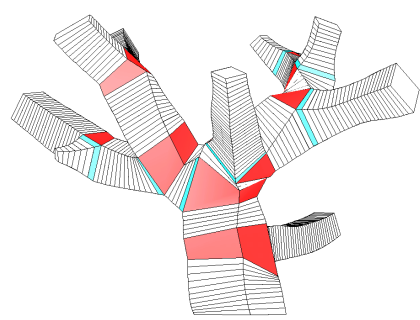

(c)

Figure 1: (a) Branches of a vascular tree (coloured by increasing parent vessel order: black, red, green, blue), (Section 2.1.1). Bifurcation normals (white) and projected bifurcation normals (coloured by parent vessel order), (Section 2.1.2). (b) Squares positioned along the branches (Section 2.1.2). (c) Multi-block structure of the vascular tree with the curved block structures in white, the branch connection blocks in red and the vessel connection blocks in cyan (Section 2.1.3).

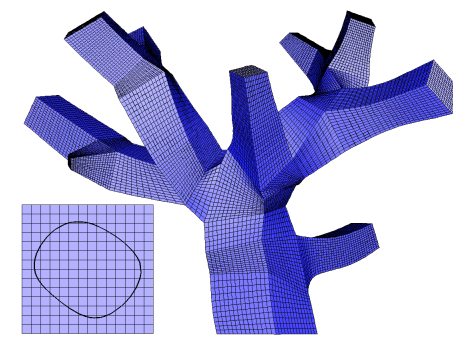

(a)

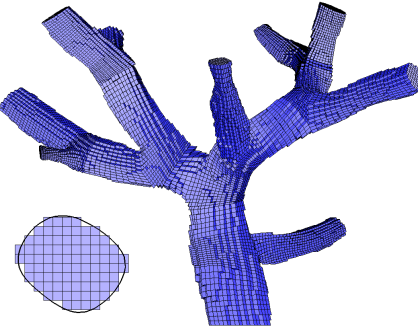

(b)

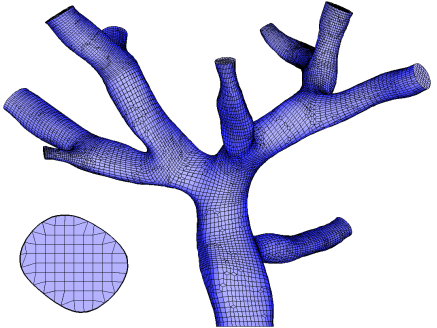

(c)

Figure 2: (a) Refined multi-block structure, of the vascular tree (Section 2.2.1). (b) and (c) Unstructured hex-core before and after the body-fitting procedure of the grid-based method (Section 2.2.2). A two-dimensional view of the inlet is presented in the lower left corner of every subfigure. 


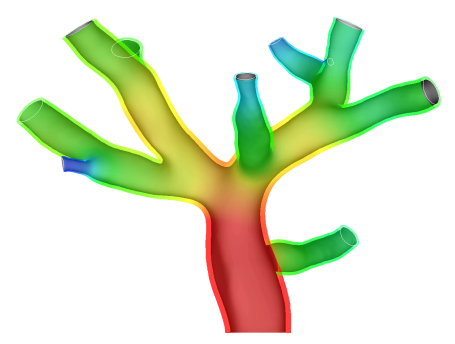

(a)

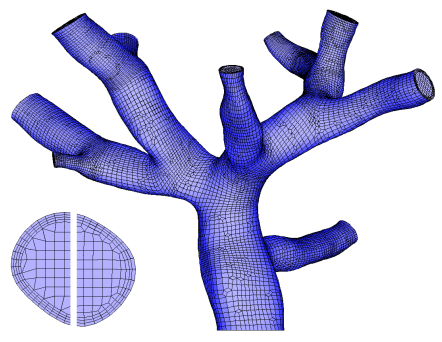

(b)

Figure 3: (a) Radially compressed interface and boundary layer region (coloured by thickness). (b) Final fluid mesh with a two-dimensional view of the inlet before (left) and after (right) smoothing. (Section 2.2.3)

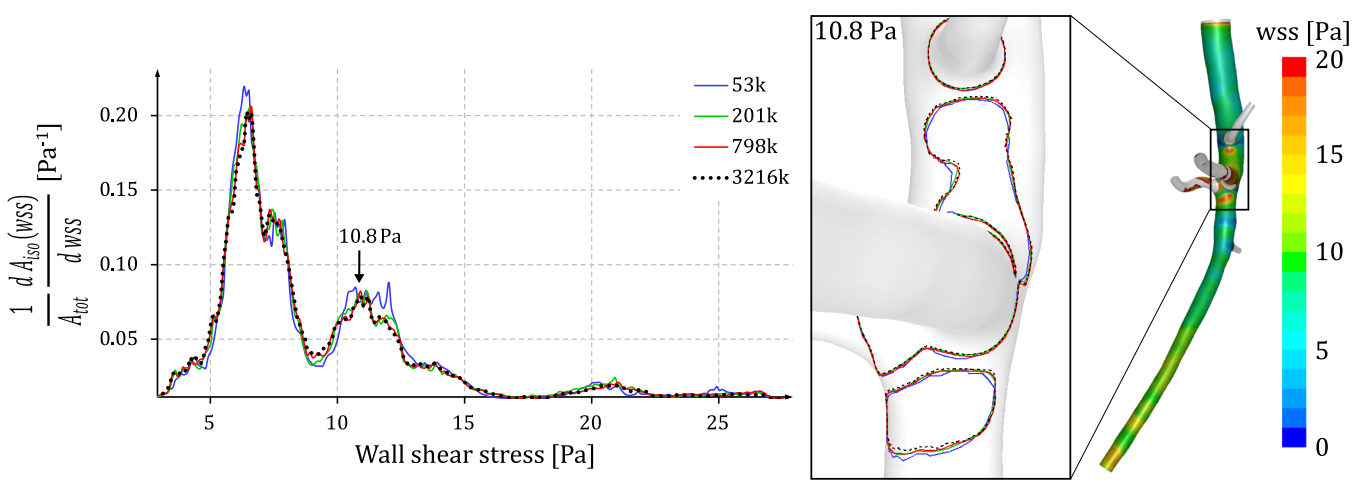

(a)

(b)

Figure 4: (a) Change in enclosed surface area in function of the wall shear stress for hexahedral grids with different mesh densities. (b) Contour plot of the wall shear stress (right) and iso-contours (left) at the trifurcation region (for the peak in wall shear stress change at $10.8 \mathrm{~Pa}$ and for different mesh densities) 


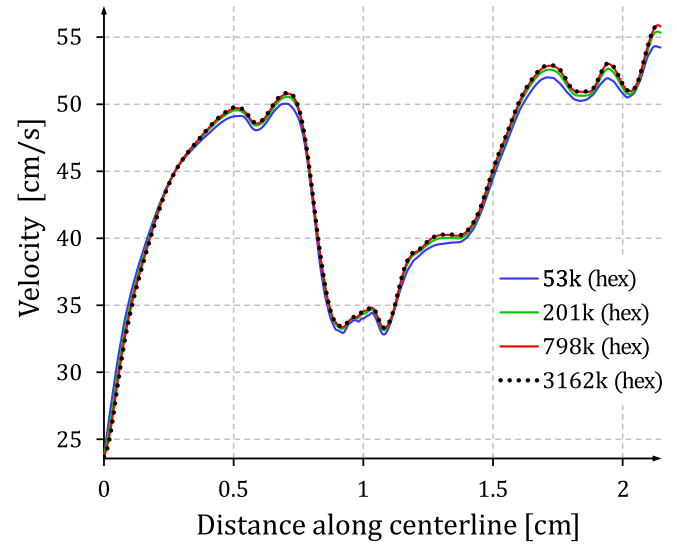

(a)

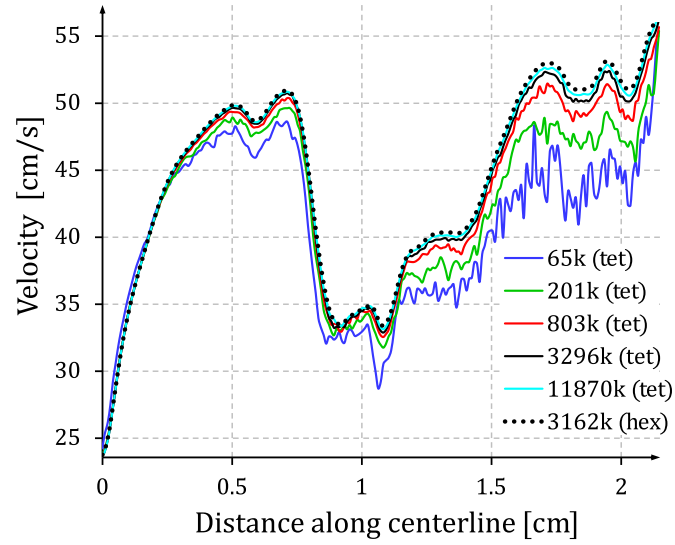

(b)

Figure 5: (a) The velocity along the centerline of the abdominal mouse aorta for hexahedral grids with different mesh densities and (b) compared to tetrahedral grids with different mesh densities. ( $0.1 \mathrm{~mm}$ between the data points) 


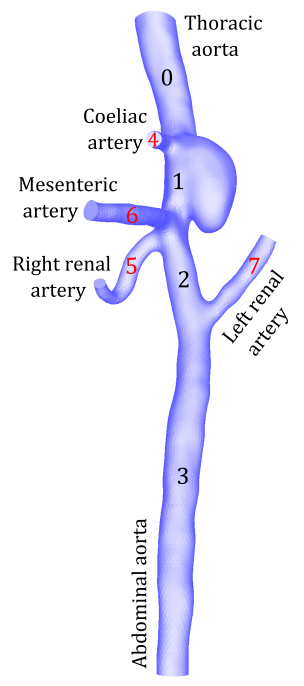

(a)

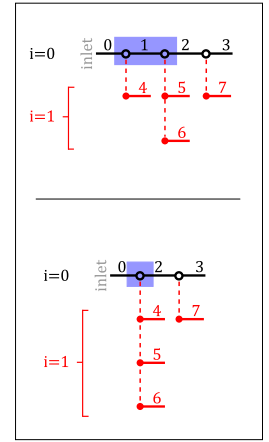

(b)

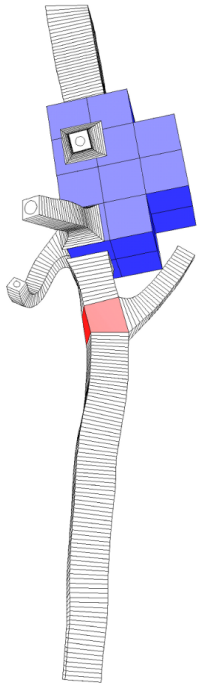

(c)

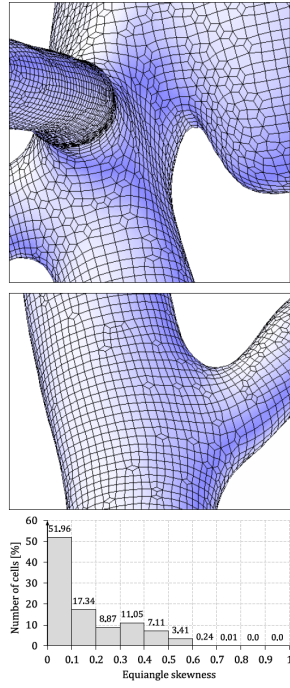

(d)

Figure 6: (a) Geometry of an abdominal mouse aorta (with aneurysm), including the branch labels (coloured by parent vessel order: i). (b) Schematic representation of the branching topology as defined in Section 2.1.1 (top) and after adaptation (bottom). (c) Multi-block structure with an adapted branch connection block comprising the aneurysm and trifurcation region (blue). (d) Detailed views of the generated fluid mesh (top, middle) and the corresponding equiangle skewness distribution (bottom). 


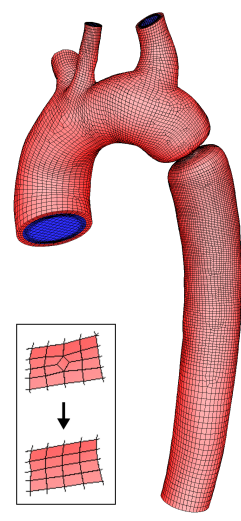

(a)
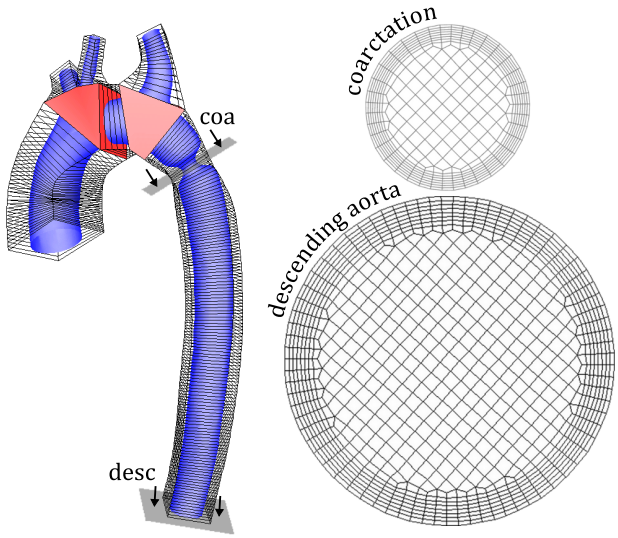

(b)
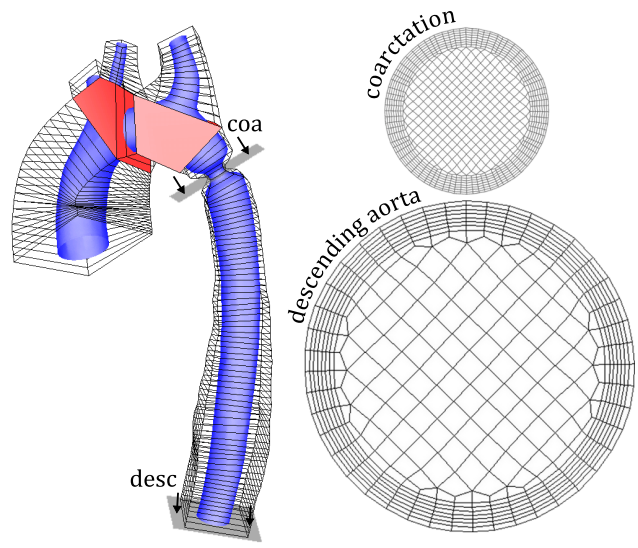

(c)

Figure 7: (a) Mesh for the fluid domain (blue) and the arterial wall (red) of an aortic arch with aortic coarctation. The lower left box demonstrates the quality improvement of the solid mesh. Note the axial coarsening towards the descending aorta (R5). (b) and (c) The cross sectional grids of the fluid mesh at the coarcation (coa) and the descending aorta (desc), which result from multi-block structures R4 (uniform grid refinement) and R5 (local grid refinement). 


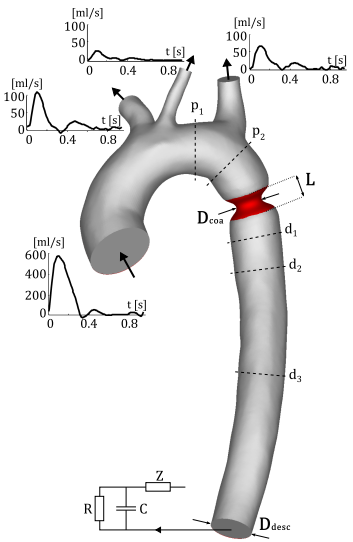

(a)

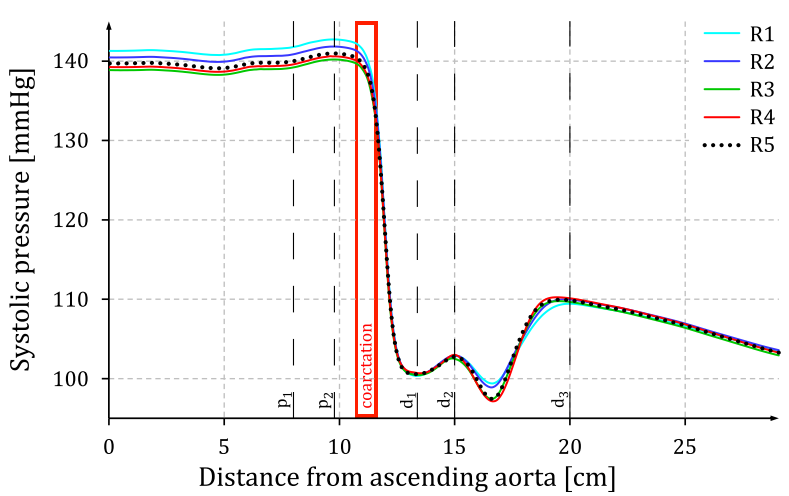

(b)

Figure 8: (a) Geometrical model of the aortic arch with aortic coarctation (red), including the boundary conditions for the fluid domain and the cross sectional regions (p1, p2, coa, $\mathrm{d} 1, \mathrm{~d} 2, \mathrm{~d} 3$, desc) in which the convergence was analyzed numerically (see Table 2). (b) Pressure along the centerline at peak systole for increasing mesh densities (R1 to R4) and a grid with a local refinement at the coarctation region and a gradual coarsening towards the descending aorta (R5). 
Table 1: Mean and maximum values of the pressure, velocity and the wall shear stress error variables for different grid densities

\begin{tabular}{|c|c|c|c|c|c|c|c|c|c|c|}
\hline \multirow{2}{*}{$\begin{array}{c}\text { \# } \\
\text { Fluid } \\
\text { cells }\end{array}$} & \multicolumn{4}{|c|}{ Pressure drop along centerline } & \multicolumn{4}{|c|}{ Velocity along centerline } & \multicolumn{2}{|c|}{ Wall shear stress area } \\
\hline & $\begin{array}{c}\Delta p \\
{[\mathrm{mmHg}]}\end{array}$ & $\begin{array}{l}e_{\Delta p} \\
{[\%]}\end{array}$ & $\begin{array}{c}e_{\text {mean }, p} \\
{[\%]}\end{array}$ & $\begin{array}{c}e_{\max , p} \\
{[\%]}\end{array}$ & $\begin{array}{c}\Delta v \\
{\left[\frac{\mathrm{cm}}{\mathrm{s}}\right]}\end{array}$ & $\begin{array}{l}e_{\Delta v} v \\
{[\%]}\end{array}$ & $\begin{array}{c}e_{\text {mean }, v} \\
{[\%]}\end{array}$ & $\begin{array}{c}e_{\max , v} \\
{[\%]}\end{array}$ & $\begin{array}{c}e_{\text {mean,wss }} \\
{[\%]}\end{array}$ & $\begin{array}{c}e_{\max , w s s} \\
{[\%]}\end{array}$ \\
\hline $53 \mathrm{k}$ & 6.877 & 0.795 & 0.151 & 0.795 & 30.528 & 0.0552 & 2.028 & 6.101 & 0.268 & 1.728 \\
\hline $98 \mathrm{k}$ & 6.853 & 0.443 & 0.171 & 0.443 & 31.106 & 0.0373 & 1.460 & 5.096 & 0.193 & 1.088 \\
\hline $201 \mathrm{k}$ & 6.844 & 0.319 & 0.0536 & 0.319 & 31.696 & 0.0190 & 0.825 & 3.174 & 0.138 & 0.827 \\
\hline $798 \mathrm{k}$ & 6.827 & 0.0638 & 0.0359 & 0.107 & 32.206 & 0.00324 & 0.186 & 1.090 & 0.0629 & 0.437 \\
\hline $1602 \mathrm{k}$ & 6.826 & 0.0449 & 0.00875 & 0.0449 & 32.267 & 0.00134 & 0.0977 & 0.428 & 0.0323 & 0.265 \\
\hline $3216 \mathrm{k}$ & 6.822 & & & & 32.310 & & & & & \\
\hline
\end{tabular}

Table 2: Grid refinement study of the pressure in an FSI model of aortic coarctation

\begin{tabular}{|c|c|c|c|c|c|c|c|c|c|c|c|}
\hline \multirow{2}{*}{ Grid } & \multirow{2}{*}{$\begin{array}{l}\text { \# Fluid } \\
\text { cells }\end{array}$} & \multirow{2}{*}{$\begin{array}{c}\text { \# Solid } \\
\text { elem. }\end{array}$} & \multirow{2}{*}{$\begin{array}{l}\text { Calc. time } \\
\text { per cycle }\end{array}$} & \multicolumn{8}{|c|}{ Mean error [\%] } \\
\hline & & & & asc & $\mathrm{p}_{1}$ & $\mathrm{p}_{2}$ & $\operatorname{coa} d_{1}$ & $\mathrm{~d}_{2}$ & $\mathrm{~d}_{3}$ & desc & \\
\hline $\mathrm{R} 1$ & $42 \mathrm{k}$ & $21 \mathrm{k}$ & $12 \mathrm{~h} 29 \mathrm{~min}$ & 1.44 & 1.42 & 1.42 & 1.72 & 1.61 & 1.56 & 1.33 & 0.77 \\
\hline $\mathrm{R} 2$ & $105 \mathrm{k}$ & $37 \mathrm{k}$ & $17 \mathrm{~h} 29 \mathrm{~min}$ & 1.01 & 0.98 & 0.98 & 1.26 & 1.54 & 1.88 & 1.26 & 0.71 \\
\hline R3 & $281 \mathrm{k}$ & $74 \mathrm{k}$ & $30 \mathrm{~h} 55 \mathrm{~min}$ & 0.38 & 0.34 & 0.34 & 0.75 & 1.22 & 1.07 & 0.94 & 0.64 \\
\hline R4 & $408 \mathrm{k}$ & $102 \mathrm{k}$ & $40 \mathrm{~h} 38 \mathrm{~min}$ & \multicolumn{8}{|c|}{ Reference grid } \\
\hline R5 & $216 \mathrm{k}$ & $51 \mathrm{k}$ & $23 \mathrm{~h} 38 \mathrm{~min}$ & 0.5 & 0.47 & 0.47 & 0.86 & 1 & 1.15 & 0.98 & 0.6 \\
\hline
\end{tabular}

